\title{
The COVID-19 pandemic threatens the Expanded Program on Immunization: recommendations for sustaining vaccination goals
}

\author{
Husnain Hamid ${ }^{1}\left[\right.$. Tauqeer Hussain Mallhi ${ }^{2} \cdot$ Muhammad Saad Naseer $^{1} \cdot$ Iqra Younas $^{1} \cdot$ Muhammad Ateeb Rashid $^{1}$. \\ Ayesha Pervaiz ${ }^{3}$ Noor ul Ain ${ }^{1}$
}

Published online: 5 September 2020

(c) Springer Nature Switzerland AG 2020

\section{Background}

Spread of the severe acute respiratory syndrome coronavirus 2 (SARS-CoV-2; COVID-19) pandemic has been unprecedented and affected daily activities at all levels. Lockdown and social distancing have been used as effective methods to halt the transmission of the disease and, ultimately, reduce morbidity and mortality. However, the impact of such measures, including the disruption of immunization schedules, is of utmost concern [1]. Children aged $<5$ years have undeveloped immune systems and are more prone to infections than other age groups. To protect children against some harmful and infectious diseases, the World Health Organization (WHO) introduced the worldwide Expanded Program on Immunization (EPI) in May 1974. Vaccination schedules are designed to attain high levels of vaccine protection in large populations [2]. It is estimated that the EPI reduced child mortality by $\approx 2-3$ million cases globally [3]. Currently, the EPI seems to be at risk because of the strained healthcare systems, lockdown policies, and disrupted immunization services caused by the COVID-19 pandemic. This commentary highlights the disruption to immunization caused by responses to the COVID-19 pandemic and proposes possible measures to improve vaccination rates and services.

With rapidly increasing vaccination coverage prior to COVID-19, it was estimated that $\approx 90 \%$ of children would be vaccinated with the Bacillus Calmette-Guérin (BCG) vaccine, the third dose of the diphtheria, tetanus, and pertussis

Husnain Hamid

husnainhamid9@gamil.com

1 Faculty of Pharmacy, University of Central Punjab, Lahore 54000, Pakistan

2 Department of Clinical Pharmacy, College of Pharmacy, Jouf University, Al-Jouf, Sakaka, Kingdom of Saudi Arabia

3 Faculty of Pharmacy, University of Lahore, Lahore 54000, Pakistan
(DTP) vaccine, the oral polio (OPV3) vaccine, and the first dose of the measles vaccine [2]. However, in a recent report (15 July 2020) [4], the WHO and UNICEF warned of an alarming decrease in vaccination rates among children. Countries responded to the sudden emergence of COVID19 in a variety of ways in terms of strategic planning. Lockdown policies disrupted healthcare facilities and services and the drug and vaccine supply chain, which ultimately decreased vaccination rates, putting children at risk for vaccine-preventable diseases (VPDs) and their complications [3].

Preliminary data for the first 4 months of 2020 from the WHO highlighted a significant drop in childhood vaccination with the three-dose DPT vaccine [4], the first time coverage with this vaccine has decreased in the past 28 years [4]. In the past decade, South-Asian countries have reported a $12 \%$ improvement in vaccination with the third dose of DTP; however, they are currently at risk of a backslide in vaccination rates [4]. In addition, 30 campaigns for measles and rubella vaccination are at risk of being cancelled, which will likely result in exaggerated outbreaks of this disease [5].

With the aim of maintaining effective immunization coverage, the WHO and UNICEF are providing global support by facilitating the delivery of essential supplies to communities and ensuring vaccination campaigns are organized during the pandemic, followed by possible infection prevention and control measures. On 25 June 2020, the WHO and UNICEF organized a 5-day campaign in Syria to rectify vaccination gaps that emerged during the COVID-19 pandemic. The vaccination status of $\approx 0.9$ million children was checked, and 0.21 million children were vaccinated [6]. In July 2020, a 4-day polio vaccination campaign was organized in Burkina Faso, and 174,304 children were vaccinated (Fig. 1) [7].

Barriers to vaccinating children vary globally. A report from UNICEF indicated that lockdown measures had significantly stalled immunization services in 68 low- and 


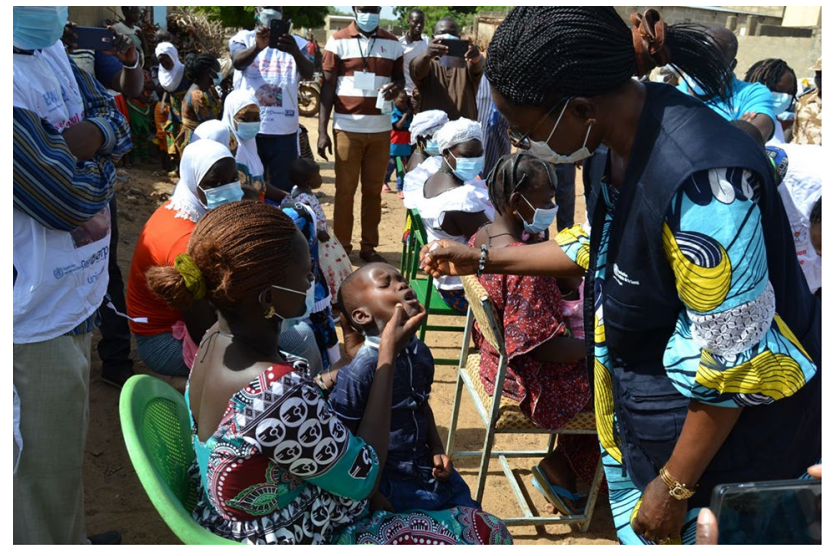

Fig. 1 Vaccination campaign in Burkina Faso as per infection prevention and control measures by the World Health Organization (WHO). Reused with permission from the WHO [7]

middle-income countries (LMICs) and placed $\approx 80$ million children aged $<1$ year at risk of VPDs [3]. Vaccination rates via services provided by national health authorities are also less than satisfactory, possibly because individuals are unwilling to leave home for fear of being infected with COVID-19, or because of transportation interruptions, economic burden, and restricted movements because of COVID-19-prevention strategies. One major barrier is the lack of healthcare professionals (HCPs) to perform vaccinations because of the burden of the pandemic, and a lack of personal protective equipment (PPE) for HCPs has also been observed [4].

\section{Recommendations}

The immunization of children is stalling, and maintaining coverage is becoming challenging, with almost 20 million children being unvaccinated or under-vaccinated in 2019 . The majority of this vaccination burden is shared by ten LMICs: Angola, Brazil, Democratic Republic of the Congo, Ethiopia, Indonesia, India, Mexico, Nigeria, the Philippines, and Pakistan [5]. The COVID-19 pandemic disrupted immunization coverage, yielding a stagnant coverage rate. Currently, there is a dire need for a collaborative approach between global and national organizations to revive disrupted vaccination rates:

- Most importantly, developed countries and international organizations should support LMICs by providing funding, vaccines, equipment, and HCPs for EPI centers.

- The WHO, UNICEF, and other alliances for vaccination coverage should ensure a definite supply of vaccines in developed and developing countries.
- International organizations should develop a surveillance system for following updates at the national level.

- Countries should prioritize immunization as an essential health service, reopen existing EPI centers and services as soon as possible, introduce policies to increase the number of EPI centers, maintain the number of HCPs at EPI centers, and provide HCPs with PPE.

- When organizing immunization campaigns, responsible departments should develop guidelines for infection prevention and control, such as managing patients in waiting rooms and increasing time slots at EPI centers.

- To rectify the vaccination gaps identified during the pandemic, an immediate response is required. Using mobile EPI centers is a dynamic and novel approach to ensure vaccine availability. EPI vans should be sent to rural and undeveloped areas to increase access to vaccines.

- Tele-health services, such as mobile phone applications and software, should be introduced, and can be used for vaccination schedules, reminders, and records, as well as to alert HCPs of children who have missed a number of vaccine doses.

- Media should support such causes by creating awareness about vaccination and providing information about nearby EPI centers.

- Pharmacies are easily accessible community-level sites for public healthcare services, and pharmacists have a potential role as vaccinators during the current health crisis. They may also educate their community about the importance of immunization and support vaccine availability and supply [8].

\section{Take home messages}

- Encourage vaccinators to play a significant role in managing childhood vaccination.

- Encourage parents to immunize their children against VPDs.

- Enable pharmacists to organize vaccine awareness campaigns at the community level and to play a distinct role in supporting EPI centers and maintaining vaccine supply.

Acknowledgements The authors acknowledge all HCPs working ceaselessly to halt the COVID-19 pandemic.

\section{Declarations}

Conflicts of interest Husnain Hamid, Tauqeer Hussain Mallhi, Muhammad Saad Naseer, Iqra Younas, Muhammad Ateeb Rashid, Ayesha Pervaiz, Noor ul Ain have no conflicts of interest that are directly relevant to the content of this article. 
Funding No sources of funding were used to prepare this manuscript.

Ethics approval, Consent to participate, Consent for publication, Availability of data and materials, Code availability Not applicable.

Author contributions All authors contributed equally to the conceptualization of this manuscript, the literature review, and the drafting, critical review, and writing of the manuscript.

\section{References}

1. World Health Organization. Guiding principles for immunization activities during the COVID-19 pandemic: interim guidance, 26 March 2020. Geneva: World Health Organization; 2020.

2. Hoest C, Seidman JC, Lee G, et al. Vaccine coverage and adherence to EPI schedules in eight resource poor settings in the MALED cohort study. Vaccine. 2017;35(3):443-51.

3. UNICEF. Immunization coverage Are we losing ground?. 2020. https://data.unicef.org/resources/immunization-coverage-are-welosing-ground/. Accessed 31 Jul 2020.
4. World Health Organization. WHO and UNICEF warn of a decline in vaccinations during COVID-19. 2020. https://www.who.int/ news-room/detail/15-07-2020-who-and-unicef-warn-of-a-decli ne-in-vaccinations-during-covid-19. Accessed 31 Jul 2020.

5. World Health Organization. Immunization coverage. 2020. https ://www.who.int/en/news-room/fact-sheets/detail/immunizationcoverage. Accessed 15 Aug 2020.

6. World Health Organization. How WHO is supporting ongoing vaccination efforts during the COVID-19 pandemic. 2020. https:// www.who.int/news-room/feature-stories/detail/how-who-is-suppo rting-ongoing-vaccination-efforts-during-the-covid-19-pandemic. Accessed 31 Jul 2020.

7. World Health Organization. Burkina Faso resumes polio vaccination campaigns under strict COVID-19 prevention measures. 2020. https://www.afro.who.int/news/burkina-faso-resumes-polio -vaccination-campaigns-under-strict-covid-19-prevention-measu res. Accessed 31 Jul 2020.

8. Hamid H, Masood RA, Khalid W, et al. Emerging pharmacy services: recommendations for emergency care of COVID-19 pandemic in low and middle-income countries. Pak J Pharm Sci. 2020;33(4):1735-8. 